\title{
Motor Ability and Emotions in Rheumatoid Arthritis Patients
}

\section{Snezana Tomasevic-Todorovic ${ }^{1 *}$, Fahad Hanna ${ }^{2}$, Ksenija Boskovic ${ }^{1}$, Danka Filipovic ${ }^{3}$, Vladimir Vidovic ${ }^{1}$ and Karmela Filipovic ${ }^{3}$}

${ }^{1}$ Clinical Center of Vojvodina, Clinic for Medical Rehabilitation, Faculty of Medicine, University of Novi Sad, Serbia

${ }^{2}$ Monash University, Department of Epidemiology and Preventive Medicine, Prahran, Australia \& Baker-IDI heart and Diabetes institute, Melbourne, Australia ${ }^{3}$ Special Hospital for Rheumatic Diseases, Faculty of Medicine, University of Novi Sad, Serbia

\begin{abstract}
Objective: The aim is to determine the influence of emotional status on motor response velocity in patients with rheumatoid arthritis.

Materials and Method: The study included 53 women with rheumatoid arthritis and 27 healthy women Depression was diagnosed using the Beck Scale for Depression (Beck Depression Inventory-BDI), anxiety using Spielberger's anxiety test (Spielberger Trait Anxiety Inventory-State and Traité), functional status (Health Assessment Questionnaire, HAQ), and disease activity using the activity index disease (DAS 28). Simple and choice reaction time was determined using a computer program Donders.

Results: Mean visuomotor reaction time (RT1, RT2, RT3) were significantly lower in the group of patients with rheumatoid arthritis than in the control group $(p<0.001)$. In the group of anxious-depressive patients was found significant differences in average values of simple and choice reaction time when compared to the pain by the VAS scale $(p<0.001)$, but not in the group of anxious patients with rheumatoid arthritis.

Conclusion: The anxious-depressive patients had damaged motor abilities, which are manifested by significantly longer simple and choice reaction time, when compared to the anxious RA patients and healthy people. The anxiousdepressive RA patients had the higher intensity of pain which correlated with longer visuomotor reaction time.
\end{abstract}

Keywords: Arthritis; Rheumatoid; Depression; Anxiety; Reaction time; Cross-sectional study

\section{Introduction}

Rheumatoid arthritis (RA) is a disease characterized by pronounced inflammatory and destructive changes in joints, causing pain, reduction of functions and motor skills of patients [1]. Multidisciplinary approach to patients with rheumatoid arthritis takes into account the complex interaction of somatic and psychological factors and opens up new vistas in understanding the mental and motoric functioning of the diseases.

Limited number of studies evaluated problems of mental functions and visuomotor abilities in patients with RA, regardless of importance, frequency and type. The heterogenity of data on the prevalence and types of emotional and cognitive impairment in patients with RA is based on the methodological differences in neuropsychological research and the diversity of clinical manifestations between patients $[2,3]$.

Numerous published data examined the impact of pain on mental functions, point to the need and importance of tests of emotional and cognitive functions in chronic pain conditions [4-7].

Studies have shown that many aspects of cognition in RA patients can be damaged $[4,8]$. Furthermore, Dick and colleagues have found that patients with RA, fibromyalgia syndrome, and musculoskeletal pain had worse performance in tests of attention, and indicated the importance of pain in attention deficiency [4]. On the other hand, Brown and colleagues examined the relationship of cognitive abilities, depression and pain in patients with RA, and found indirect connection between pain, cognition and depression [9]. Other data indicate an association between peripheral levels of cytokines, hormone status and cognitive dysfunction [10].

Data obtained by simple and complex psychometric measurements in patients with rheumatoid arthritis show the impact of pain on mental functioning, and provide opportunities for developing new therapeutic strategies [5,6]. The connection of disturbed mental functioning and the velocity of motor response in patients with rheumatoid arthritis creates the need for an additional clinical neurophysiological diagnostics $[11,12]$.

These diagnostics in patients with RA, as well as in other chronic pain conditions, should incorporate an additional method of examining the various reaction times of visual-motoric type [12]. Reaction time is the time needed to respond to specific stimulation by adequate usually motor response. The simple reaction time is the time interval from the occurrence of stimulus to the development of responses to a given stimulus. The choice reaction time-(CRT) involve the time required for the differentiation offered a number of stimuli, and the time required for the selection of responses to given stimuli. Choice reaction time is a kind of complex reaction time. The choice reaction time is more sensitive than simple reaction time and more susceptible to the influences of various factors [13]. The usage, of the simple and choice reaction time may help examine cognitive abilities of patients.

Obviously, the only optimal approach to patients with rheumatoid arthritis is individual and multidisciplinary, in order to consider interactions between their pain, their inflammation, patient's mental functions and their motor abilities.

*Corresponding author: Snezana Tomasevic-Todorovic, Faculty of Medicine University of Novi Sad, Serbia, Tel: 0038163540524 ; E-mail: drtomasevic@gmail.com

Received August 21, 2012; Accepted September 28, 2012; Published September 30, 2012

Citation: Todorovic ST, Hanna F, Boskovic K, Filipovic D, Vidovic V, et al. (2012) Motor Ability and Emotions in Rheumatoid Arthritis Patients. J Neurol Neurophysio 3:137. doi:10.4172/2155-9562.1000137

Copyright: (c) 2012 Todorovic ST, et al. This is an open-access article distributed under the terms of the Creative Commons Attribution License, which permits unrestricted use, distribution, and reproduction in any medium, provided the original author and source are credited. 
Objective: The aim of this study was to determine the influence of emotional status on motor responce velocity in patients with rheumatoid arthritis using the method of examination of the various reaction times of visual-motoric type.

\section{Materials and Methods}

The study included 80 subjects who were introduced with research methods previosly. The RA group consisted of 53 women with mean age of $50.58 \pm 0.93$ years, and who fulfilled the criteria for the diagnosis of the rheumatoid arthritis based on the American Rheumatology Association (ARA) in 1987. The research was done in the Special Hospital for Rheumatic Diseases, and in Neurophysiological laboratory -Department of Physiology, Medical Faculty in Novi Sad, Serbia during 2009. The CO group (control group) of 27 healthy women with mean age of $49.41 \pm 1.08$ years, was used for setting a normative of neuropsychological parameters. Each participant was given detailed information on how to prepare for the test and the type of applied tests and tasks. Exclusion criteria included: hearing impairment, dementia, neurological and psychiatric diseases, head trauma, use psychoactive substances, antidepressants three weeks before the survey or surgery a month before the survey.

In order to achieve the objectives of the research we used anamnesis data, clinical examination, and questionnaires. In the RA group the following parameters were collected: number of painful and swollen joint count, pain in the joints of patients evaluated by a visual analogue scale (VAS) of 0-100 mm, speed sedimentation rate, an index of disease activity. The examination of functional abilities of patients was performed with the Health Assesment Questionnaire (HAQ)involving 20 questions from 8 functional categories. We used index Disease Activity Score (DAS 28) to estimate the disease activity.

Tests for evaluation of emotional manifestations in patients with rheumatoid arthritis were conducted in the Special Hospital for Rheumatic Diseases in Novi Sad.

Spielberger's anxiety test (Spielberger Trait Anxiety InventoryState and Traité) was used for detection of anxiety, which consists of two questionnaires (STAI-S, STAI-T) with 20 claims for standardized measurement of current and general anxiety. A total score of 30 points reflects a low level of anxiety, while a score 31-44 reflects a moderate level, and scores above 45 is indicative of high level of anxiety.

For evaluation of depression Beck's depression scale (Beck Depression Inventory-BDI) was applied, covering 21 aspects of depression. BDI total score determines the weight of depression, and scores above 30 indicate severe clinical depression.

Reaction time of patients in relation to three types of tasks, was determined using a computer program Donders. The task was to press the appropriate key on the keyboard of the computer as quickly as possible, in response to $\mathrm{X}$ sign at the computer screen. The task and the possible locations of stimulus were different depending on the block attempt.

The first type of task, so. one stimulus-one motor response, involves the appearance of stimuli on the right fixed point of the screen in each trial, has served for the determination of simple motor reaction time. In the second block of tasks RT2, so. two stimulus-one response, the stimulus can appear on both sides of the screen (left or right), however, the subject was instructed to press the button on the keyboard with his index finger when the stimulus appears only on the right fixed point. In the third block of tasks RT3 two visual stimuli-two motor responses two stimuli can occur in the two different positions on the screen, and the respondent should respond by pressing appropriate keys on both the stimuli. Using second and third block of tasks is determined by a choice visuomotor reaction time. Each block of tasks consist of twenty trials with an interval between each attempt of $500 \mathrm{~ms}$. Raw data obtained by measuring the reaction time were analyzed using DONDANAL that calculates the arithmetic mean and standard deviation of reaction time.

Prior to coming to research at Neurophysiological laboratory, at the Faculty of Medicine in Novi Sad, the examinees did not take any psychoactive substances (such as coffee, tea, alcohol) and had enough sleep thus were rested. During the research, the examinees were placed a comfortable chair, in a pleasant interior, two hours after a meal, on days when they did not receive disease modifying drugs. Each participant receives detailed information on how to solve tasks and has the opportunity to try to resolve them through a test series containing all sorts of tasks. The procedure of determining the reaction time lasted 25-30 minutes. An examination protocol was specially designed for all participants of the study.

Prior to testing subjects reaction time grip strength was measured using a dynamometer in order to eliminate the influence of grip strength on the results of reaction time. The study was approved by the Ethics Committee of the Clinical Center of Vojvodina and Faculty of Medicine, University of Novi Sad.

\section{Statistical Methods}

For the purpose of statistical analysis, the measures of descriptive statistics - frequency, mean, measures of variation were utilised. In the paper, parametric (t-test) and nonparametric (Mann Whitney test, chisquare test) were used to compare the distribution of two groups. To compare three or more groups of respondents the analysis of varianceANOVA and Kruskal-Wallis test was used. Correlation of continuous variables was examined by Pearson correlation coefficient.

\section{Results}

The RA group consisted of 53 patients with rheumatoid arthritis, while the CO group consisted of 27 healthy subjects who were not significantly different from RA group in regards to age, education level, marital status - the relevant characteristics of the applied methods ( $\mathrm{CV}<30$ ) (Table 1).

Results of the emotional disturbance detection using Beck's Depression Inventory (BDI) and Spielberger's anxiety test showed both signs of depression and anxiety in 35 patients $(66.04 \%)$, and the presence of anxiety symptoms in $18(33.96 \%)$ patients, as shown on the attached tables (Table 1). Under CO, 7 (14.82\%) subjects showed healthy mild symptoms of depression and anxiety, while $20(85.18 \%)$ showed the presence of anxiety symptoms (Table 1).

Average score of depression obtained by Beck's Depression Inventory (BDI) was in the $\mathrm{CO}$ group $7.41 \pm 0.84$, and $18.15 \pm 1.66$ in the RA group. There was a statistically significant difference of the values of depression scores between the 2 groups $(\mathrm{p}<0.001)$ (Table 1$)$.

Average scores obtained by Spielberger's anxiety test (STAI-S) that measures state anxiety were in the group CO $40.77 \pm 1.63$ and in the RA group $49.77 \pm 0.94$. There was a statistically significant difference in the values of state anxiety scores between the 2 groups (Table 1 ).

Average scores obtained by Spielberger's anxiety test (STAI-T) that measures general anxiety, in the group CO were $41.67 \pm 1.59$ and in the RA group $53.83 \pm 0.76$. There was a statistically significant difference in 


\begin{tabular}{|c|c|c|}
\hline General characteristics & Group CO & Group RA \\
\hline Number & 27 & 53 \\
\hline Age (years), $\mathrm{X} \pm \mathrm{SD}$ & $49,41 \pm 1,08$ & $50,58 \pm 0,93$ \\
\hline \multicolumn{3}{|l|}{ Degree (N) } \\
\hline lower & 9 & 20 \\
\hline medium & 12 & 27 \\
\hline higher & 2 & 4 \\
\hline high & 4 & 2 \\
\hline \multicolumn{3}{|l|}{ Marital status $(\mathrm{N})$} \\
\hline yes & 23 & 47 \\
\hline no & 1 & 2 \\
\hline other & 3 & 4 \\
\hline \multicolumn{3}{|l|}{ Affective status (N) } \\
\hline anxious-depressive & 7 & 35 \\
\hline anxious & 20 & 18 \\
\hline$B D I, X \pm S D$ & $7,41 \pm 0,84 \dagger$ & $18,15 \pm 1,66 \dagger$ \\
\hline STAI-S, $X \pm S D$ & $40,77 \pm 1,63 t$ & $49,77 \pm 0,94 t$ \\
\hline STAI-T, $X \pm S D$ & $41,67 \pm 1,59 \dagger$ & $53,83 \pm 0,76 t$ \\
\hline
\end{tabular}

* SD-standard deviation, BDI-Beck Depression Inventory, STAI-S-state-trait anxiety inventory: state subscale, STAI-T State-Trait Anxiety Inventory: Trait subscale, $\dagger$ statistically significant difference between the groups $(p<0,001)$.

Table 1: Demographic characteristics of the CO group and the RA g.

the values of general anxiety scores between the 2 groups $(p=0.00009)$ (Table 1).

The results of tests of functional ability of patients using a questionnaire Health Assessment Questionnaire (HAQ) shows average values of $0.92 \pm 0.09$ (Table 2). The average disease activity was $4.98 \pm$ 0.12 (Table 2). The table 2 shows the high level of average value of pain intensity determined with VAS scale.

The average values of simple reaction time (RT1) in the control group was $367.21 \pm 13.34 \mathrm{~ms}$, and in the RA patients was $429.69 \pm 10.63$ ms. A statistically significant difference was found between the average length of simple reaction time (RT1) between healthy subjects and RA patients $(\mathrm{p}<0.001)($ Table 3$)$.

In the second block of tasks the average length of reaction time (RT2) in the control group was $413.41 \pm 11.62$, while among patients with rheumatoid arthritis $486.97 \pm 10.27 \mathrm{~ms}$, indicating a statistically significant difference $(\mathrm{p}<0,001)$ (Table 3$)$.

The results obtained in the third block of tasks-RT3 show a significant difference in the average length of reaction time between patients with rheumatoid arthritis and healthy people $(\mathrm{p}<0.05)$ (Table $3)$. It was observed that with increasing task difficulty increases the difference in the speed of visuomotor reaction between the 2 groups.

A statistically significant prolongation of the average value of reaction time (RT1, RT2, RT3 ) was found in the group of anxiousdepressive patients when compared to anxious RA patients $(\mathrm{p}<0.05)$ (Figure 1).

The test results correlation values of reaction time with indicators of disease activity showed high positive correlation with the intensity of pain in a group of anxious-depressive patients $(\mathrm{p}<0.05)$ (Figure 2,3 and Table 4).

In the group of anxious-depressive patients were found highly significant differences in average values of simple and choice reaction time compared to the pain by the VAS scale $(\mathrm{p}<0.001)$, but not the group of anxious patients with rheumatoid arthritis.

In the group of anxious patients were found positive linear correlation between mean duration of disease and the value of reaction time (RT1, RT2, RT3), which was statistically significant for the values of choice reaction time $(\mathrm{RT} 3)(\mathrm{p}<0.05)($ Table 4$)$.

\section{Discussion}

Motor abilities testing in patients with rheumatoid arthritis is of great importance in monitoring the effects of treatment, as well as in various activities of daily life such as physical activity.

To assess mental functioning, specially emotions, i.e. efficiency of brain information processing in patients with rheumatoid arthritis, a method of visuomotor reaction time was utilized [14]. Our study shows that mental chronometry, by using the visuomotor reaction time for the development of the theory of information processing, helps clarify the mental processes underlying the perception, attention, memory and action, and that it is strongly connected with emotional states [15]. The method of reaction time is referred to as „micro-behavioral analysis" or analysis of flow parameters of motor responses [16].

Simple visuomotor reaction time, the reaction to the familiar stimulus and the same response is expected in all further attempts, and the candidate has opportunity to improve programming movement. In a complex reaction-time nature of the response is not known in advance and therefore no benefits associated with anticipation. The choice reaction time, which includes the analysis of stimulus and response selection ( i.e., central cognitive processing), is strongly influenced by emotional status.

Data concerning tests of mental and motor abilities in patients with rheumatoid arthritis using the method of reaction time are scarce. One study by Kauranen et al. used the multi-functional computer system (HPM / BEP, version 4.2) designed to test motor abilities [11]. Kauranen et al. found no significant correlation between tests of motor ability and pain intensity by visual analogue scale, duration of illness and number of swollen joints [11].

The results of our tests of reaction time shows a significant difference in the first block of tasks between the average length of simple reaction time in the group of CO and of RA group. People with rheumatoid arthritis had a significantly longer simple reaction time compared to healthy people.

In the second block of tasks, the average length of reaction time in the group of CO was $397.89 \mathrm{~ms}$. and $476.91 \mathrm{~ms}$. in the RA group, indicating a statistically significant difference. In the third block of tasks results shows a significant difference in the average length of reaction time (RT3) between patients with rheumatoid arthritis and healthy subjects, which is consistent with Kauranen work [11]. People

\begin{tabular}{|c|c|}
\hline Indicators of disease activity & Average values \pm SD \\
\hline Duration of illness/yrs/ & $9,28 \pm 7,96$ \\
\hline DAS28 & $4,93 \pm 0,85$ \\
\hline HAQ & $0,88 \pm 0,67$ \\
\hline Intensity of pain VAS & $55,33 \pm 20,95$ \\
\hline
\end{tabular}

*DAS28-disease activity score 28, HAQ-Health Assessment Questionnaire, VASvisual analogue scale, yrs-years

Table 2: The average values of indicators of disease activity.

\begin{tabular}{|c|c|c|c|}
\hline Reaction time & Group CO X \pm SD & Group RA X $\pm S D$ & $P$ values \\
\hline RT1 & $367.21 \pm 13.34$ & $429.69 \pm 10.63$ & $p<0,001$ \\
\hline RT2 & $397,89 \pm 52,97$ & $476,91 \pm 81,27$ & $p<0,001$ \\
\hline RT3 & $412,63 \pm 53,86$ & $492,43 \pm 84,64$ & $p<0,001$ \\
\hline
\end{tabular}

Table 3: The average values of reaction time in the $\mathrm{CO}$ group and the RA group. 


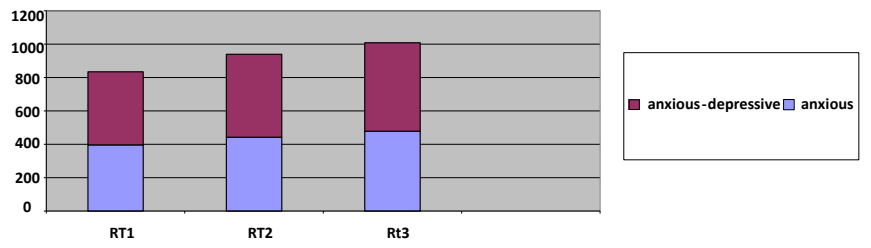

Figure 1: The average values of reaction time in the group of anxious and anxious-depressive RA patients.
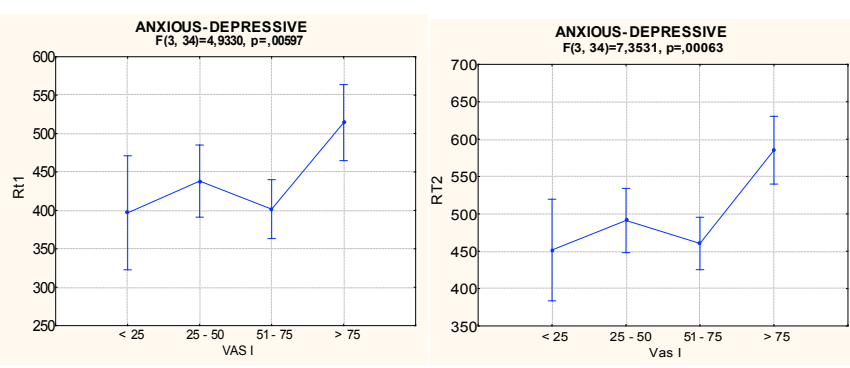

Figure 2: The connection between the values of reaction time(RT1, RT2) and the pain intensity in the group of anxious-depressive RA patients.

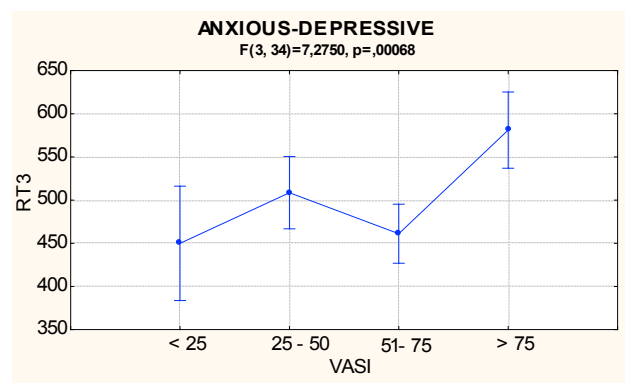

Figure 3: The connection between the values of reaction time(RT3) and the pain intensity in the group of anxious-depressive RA patients.

\begin{tabular}{|l|c|c|c|c|c|c|}
\hline \multirow{2}{*}{ Indicators of disease activity } & \multicolumn{3}{|c|}{ Anxious -depressive } & \multicolumn{3}{c|}{ Anxious } \\
\cline { 2 - 7 } & RT1 & RT2 & RT3 & RT1 & RT2 & RT3 \\
\hline Duration of illness & 0,12 & 0,17 & $-0,02$ & 0,41 & 0,35 & 0,58 \\
\hline DAS 28 & $-0,29$ & $-0,20$ & $-0,13$ & 0,14 & 0,06 & 0,15 \\
\hline HAQ & 0,09 & 0,16 & 0,25 & $-0,21$ & $-0,30$ & $-0,09$ \\
\hline VAS I & 0,26 & 0,37 & 0,32 & 0,00 & $-0,05$ & 0,06 \\
\hline
\end{tabular}

Table 4: The correlations of indicators of disease activity with the reaction time.

with rheumatoid arthritis were significantly slower to react in a block of tasks one of two stimulus-response compared to healthy people. It is known that the motor reaction is slower if the demands that are placed before the respondents are complex [17-19].

It was observed that with increasing task difficulty the difference in the speed of reaction between the CO group and the RA group also increases. This is consistent with research data obtained in patients with chronic pain $[3,6]$. It is believed that chronic pain, and fear of pain, as well as destructive and inflammatory changes in joints of RA patients together contribute to the reason for poor achievement on tests of reaction time $[11,18]$.

We found a statistically significant difference in mean values of visuomotor reaction time in the RA group between the anxiousdepressive and anxious patients. Anxious-depressive patients with rheumatoid arthritis were inhibited and slower to react than the anxious patients. Similar results are found in the work of Taimei and associates in patients with chronic back pain, which showed longer reaction time [12].

There is a statistically significant difference in approximate values of simple and choice reaction time when compared to the pain intensity, but this is not the case in the anxious group of patients. Another research was done and it showed the connection between the values of visuomotor reaction time and the indicators of disease activity. The research showed that there was the positive correlation between the reaction time and the pain intensity found in the group of anxiousdepressive RA patients. These results are consistent with the model of mental functioning proposed by Brown and associates [9].

Our results showed a significant high positive correlation between the average values of visuomotor choice reaction time and the disease duration found in the group of anxious RA patients.

\section{Study Limitations}

Further research should be aimed at improving and standardizing the methods of reaction time in daily clinical work with patients with rheumatoid arthritis, in combination with other neuropsychological and neurophysiological testing methods. Extension of studies and longitudinal monitoring of disease activity, neuropsychological, neurophysiological parameters in patients with rheumatoid arthritis depending on the hormonal phase in women of childbearing period and during menopause, would contribute to better results. Our results are preliminary, and should be tested in a higher number of patients both gender.

\section{Conclusions}

Based on our research, we can conclude that the anxious-depressive patients had significantly longer simple and choice reaction time, when compared to the anxious RA patients and healthy people. The anxious-depressive RA patients had the higher intensity of pain which correlated with longer visuomotor reaction time. The anxious patients had the correlation between the length of reaction time and the disease duration.

\section{References}

1. Katz WA(1998) Rheumatoid arthritis. Diagnosis and Management of Rheumatic Diseases. Philadelphia: Lippincott 380-96.

2. Tomasević-Todorovic S, Bosković K, Filipović D, Naumović N (2011) Assessment of memory in patients with rheumatoid arthritis. Vojnosanit Preg 68: 481-488.

3. Grisart JM, Plaghki LH (1999) Impaired selective attention in chronic pain patients. Eur J Pain 3: 325-333.

4. Dick B, Eccleston C, Crombez G (2002) Attentional functioning in fibromyalgia rheumatoid arthritis, and musculoskeletal pain patients. Arthritis Rheum 47: 639-644.

5. Ellis HC, Ottaway SA, Varner LJ, Becker AS, Moore BA (1997) Emotion, motivation, and text comprehension: the detection of contradictions in passages. J Exp Psychol Gen 126: 131-146.

6. Veldhuijzen DS, Kenemans JL, van Wijck AJ, Olivier B, Kalkman CJ, et al (2006) Processing capacity in chronic pain patients: a visual event-related potentials study. Pain 121: 60-68.

7. Tomasevic-Todorovic S, Filipovic D, Boskovic K (2010) The effect of pain on cognitive abilities of patients with rheumatoid arthritis. Clinical Neurophysiology 121: 18.

8. Hanly JG, Fisk JD, McCurdy G, Fougere L, Douglas JA (2005) Neuropsychiatric syndromes in patients with systemic lupus erythematosus and rheumatoid arthritis. J Rheumatol 32: 1459-1456. 
Citation: Todorovic ST, Hanna F, Boskovic K, Filipovic D, Vidovic V, et al. (2012) Motor Ability and Emotions in Rheumatoid Arthritis Patients. J Neurol Neurophysiol 3:137. doi:10.4172/2155-9562.1000137

Page 5 of 5

9. Brown SC, Glass JM, Park DC (2002) The relationship of pain and depression to cognitive function in rheumatoid arthritis patients. Pain 96: 279-284.

10. Kozora E, Thompson LL, West SG, Kotzin BL (2001) Analysis of cognitive and psychological deficits in systemic lupus erythematosus patients without overt central nervous system disease. Arthritis \& Rheumatism 39:2035-2045.

11. Kauranen K, Vuotikka P, Hakala M (2000) Motor performance of the hand in patients with rheumatoid arthritis. Ann Rheum Dis 59: 812-816.

12. Taimela S, Osterman K, Alaranta H, Soukka A, Kujala UM (1993) Long psychomotor reaction time in patients with chronic low-back pain: preliminary report. Arch Phys Med Rehabil 74: 1161-1164

13. Ginsburg KS, Wright EA, Larson MG, Fossel AH, Albert M, et al. (1992) A controlled study of the prevalence of cognitive dysfunction in randomly selected patients with systemic lupus erythematosus. Arthritis Rheum 35: 776-782.

14. Rizzolati G. (1986) Reaction times and mental states International Conference an; Emotions and Hemispheric specialization. Rome.
15. Umilta C. (1988) The control operations of consciousness. U: Marcel AJ. and Bisiach E.(eds). Consciousness in Contemporary science, Clarendon Press. Oxford. 334-356

16. Vrtunski PB (1982) Uticaj kognitivne težine zadatka i starosti na dinamiku izbornog reagovanja. Revija za psihologiju 15:1-2.

17. Eysenck M (1987) Anxiety and cognition: A Unified Theory, Essays in Cognitive Psychology Series.

18. Baker NA, Rogers JC (2010) Association between computer use speed and age, impairments in function, and touch typing training in people with rheumatoid arthritis. Arthritis Care Res (Hoboken) 62: 242-250.

19. Welford AT (1986) Practice effects in relation to age: a review and a theory Dev. Neuropscyhol 1:173-190. Note on the effects of practice on reaction times. J Mot Behav 18:343-345. 